\title{
Correspondence
}

Archives of Disease in Childhood, 1973, 48, 746.

\section{Development of visual acuity}

Sir,

I was interested in 'Development of Visual Acuity' by Catford and Oliver (Archives, January 1973, 48, 47). They appear to relate the size of an object which will produce optokinetic nystagmus with a similar sized Snellen letter and report visual acuities based on these findings. Sheridan (1973) has pointed out, however, that it is necessary to distinguish between the 'minimum observable' object and the 'minimum separable' object. Any attempt to equate balls and Snellen letters must consider a ball's linear diameter in relation to a letter's line thickness, i.e. to $1 / 5$ th of the width of the Snellen framework square. From the figure it looks as if Catford and Oliver are using solid circles, which would in this respect be similar to the balls used by Sheridan, to initiate nystagmus.

It is not clear whether they have considered the problem outlined above. If not, the acuities they report are inaccurate. In any case it is doubtful how wise it is to equate one testing procedure with another (letter discrimination and optokinetic nystagmus).

As a clinical tool I personally feel there are limitations to the drum and advantages to the Sheridan Stycar Tests. By 3 years of age all except handicapped children are easily tested with the 5-letter card letter matching test and I have personally tested many $2 \frac{1}{2}$-year-olds successfully with this test. I expect to find visual acuity of $6 / 6$ at this age.

At 2 years of age the child can be tested with small toys and if he can discriminate between the small knife and fork at $\mathbf{1 0}$ feet he shows not only reasonable visual acuity (it is again probably unreasonable to attempt to relate this to a Snellen visual acuity), but also that he is looking at objects in a meaningful way. At earlier ages the fixed or rolling balls are invaluable and I find it easy to get 6-month-old babies to fixate and follow the $1^{\prime \prime}$ ball (testing takes about 30 seconds). I have not so far seen many babies who failed this test, so it will be some time before one can relate findings to later visual acuity. The advantages of using the graded balls test over Catford and Oliver's optokinetic drum seem to me to be that one is testing normal, everyday function and observing meaningful use of the child's visual apparatus.

I understand that a paper reporting on some of Sheridan's own findings has recently been accepted by the editors of Developmental Medicine and Child Neurology.

MARTIN C. O. BAX Guy's Hospital, London SE1 9RT.
REFERENCE

Sheridan, M. D. (1973). The Stycar graded-balls vision test. Developmental Medicine and Child Neurology, 15, 423.

We showed this letter to Mr. Catford, who replied:

I am grateful to Dr. Bax for his comments. In reply to these, the nystagmus drum uses the following movement of fixation with the minimum observable, giving a dynamic visual acuity result. A static acuity using the minimum separable was not invoked in the test (and certainly, without the observable, separable would not be possible).

The target sizes were equated to produce an angle of 5 seconds of arc at 2 feet from the eye; Snellen nomenclature was used for relative comparison and because this is understood by physicians and administrators alike.

The drum dots have been correlated with Sheridan spheres, Sheridan Gardiner letters, and Snellen types. Accuracy has been shown between the dots and the other targets, which is the requirement of the clinician in assessing the management and educational needs of children from one examination to another.

The advantage of the drum is that results may be obtained in young children ( 6 weeks to 18 months), and also in retarded and handicapped children who will not respond to other tests. As the apparatus is used at 2 feet, it has a versatility in difficult and unusual circumstances which is not possible with more cumbersome equipment, and it is easily and quickly used.

It is acknowledged that neither the graded spheres nor the drum is a complete answer, but they may complement one another until further advances in methods of investigation are made.

G. V. CATFORD 6 Devonshire Place, London $W 1$.

\section{Abnormal mucosa in gastroenteritis}

Sir,

We read with interest the observations of Barnes and Townley in the Archives (1973) concerning the mucosal abnormalities found in the duodenum on biopsy of 26 out of 31 infants with acute gastroenteritis. This is of interest in relation to the well recognized finding of mucosal abnormalities on biopsy of children with postgastroenteritis malabsorption, e.g. 13 children out of a total of 20 biopsied with this syndrome in Sydney were 


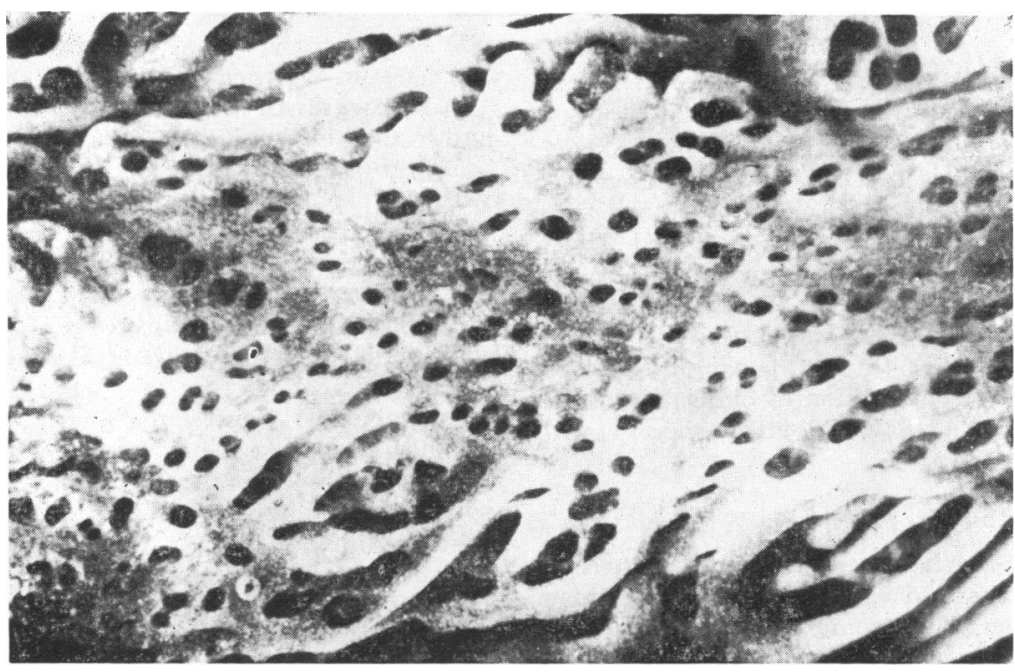

FIG.-Duoderal mucosa showing flat appearance with operings of crypts on top of a mucosal fold and short thick ridges on the sides. (Partly autolysed mucosa stained with ink. $\times 29$.)

found to have an abnormal mucosa in one series (WalkerSmith, 1967). In a more recent series of 14 children biopsied in London who had continuing diarrhoea and failure to gain weight satisfactorily 1 to 3 months after gastroenteritis, 12 had abnormal small intestinal mucosa on biopsy, 2 having a flat mucosa, and 10 having a lesser degree of abnormality (M. Rossiter, B. Wharton, and M. Boyce, personal communication, 1973). Barnes and Townley clearly documented the small intestinal mucosal abnormalities that may occur at the time of an acute attack of gastroenteritis, but the authors concluded that single biopsy specimens provide reliable information about the degree of duodenal mucosal damage on the basis of 'a consistent trend towards normality' in 3 cases that they biopsied serially. We do not feel that this is enough evidence to support their above conclusion.

Using a modification of the method of Creamer and Leppard (1965) to study the small intestine in children at $\mathrm{n}$-cropsy, a study has been reported of the uniformity of dissecting microscope appearances in the area of the proximal small intestine usually biopsied (Walker-Smith, 1972a). In 24 out of 25 children who had an abnormal small intestinal mucosa there was a significant variation of morphology within the biopsy area, i.e. the lesion was this patchy in its severity.

Eight of the children had acute enteritis or enterocolitis, none had cosliac disease. The Fig. illustrates this variability of mucosal damage within a small area.

In a more detailed report of 10 children who died from enteritis or enterocolitis (Walker-Smith, 1972b), using the above technique it was found that 7 had abnormal mucosa and that the severity and distribution of the mucosal abnormality along the small intestine was very variable. The site of maximal pathology varied from a chiefly proximal to a chiefly distal lesion or, as occurred in one child, to an equal abnormality of the mucosa along the length of the small intestine.

These findings in enteritis in children contrast with those found in an adult with coeliac disease reported by Creamer and Leppard (1965) who found at necropsy that in the most proximal part of the small intestine the mucosa was uniformly flat, though the abnormality became less severe along the length of the small intestine where variation at any one level became greater.

The observation of variable extent and severity of pathology along the small intestine in children (damage to the mucosa occurring as a sequel to gastroenteritis) explains the lack of correlation both between the severity of the biopsy abnormality and the clinical status, and also between disaccharidase deficiency shown on biopsy and clinical sugar malabsorption, as in the report of Barnes and Townley.

\section{JOHN WALKER-SMITH and MARY ROSSITER Queen Elizabeth Hospital for Children, Hackney Road, London E2 8PS.}

\section{REFERENCES}

Barnes, G. L., and Townley, R. R. W. (1973). Duodenal mucosal damage in 31 infants with gastroenteritis. Archives of Disease in Childhood, 48, 343.

Creamer, B., and Leppard, P. (1965). Post-mortem examination of a small intestine in the coeliac syndrome. Gut, 6, 466.

Walker-Smith, J. (1967). Dissecting microscope appearance of small bowel mucosa in children. Archives of Disease in Childhood, 42, 626.

Walker-Smith, J. A. (1972a). Uniformity of dissecting microscope appearances in proximal small intestine. Gut, 13, 17

Walker-Smith, J. (1972b). Small intestinal morphology in childhood. Arquivos de Gastroenterologia, 9, 119. 